\title{
Protection of Microgrids With High Amounts of Renewables: Challenges and Solutions
}

\author{
Di Liu \\ Department of Electronic and \\ Electrical Engineering \\ University of Strathclyde \\ Glasgow, UK \\ d.liu@strath.ac.uk
}

\author{
Dimitrios Tzelepis \\ Department of Electronic and \\ Electrical Engineering \\ University of Strathclyde \\ Glasgow, UK \\ dimitrios.tzelepis@strath.ac.uk
}

\author{
Adam Dyśko \\ Department of Electronic and \\ Electrical Engineering \\ University of Strathclyde \\ Glasgow, UK \\ A.Dysko@strath.ac.uk
}

\begin{abstract}
Microgrid is a small-scale network including generators, loads and storage system, which provides a friendly way for the penetration of renewables and releases the burden of transmission system arising from the increased energy demand. Moreover, since microgrid can operate in islanded mode, it can provide backup power to local consumers when the main grid is disconnected. However, the utilization of microgrid causes serious problems in the area of power system protection. The main issues comprise varied fault levels in different operating modes and fault detection in islanded microgrid particularly when the microgrid is dominated by inverter based DGs (IIDGs). In addition, to avoid non-necessary power losses raised from multi-stage power conversion of DC loads and generators, DC microgrid becomes another attractive choice, which further increases the difficult on designing protection system for the futuristic microgrid. In this paper, a comprehensive review of the existing issues and protection methods for AC and DC microgrids is presented. Furthermore, to facilitate better understanding to readers, the benefits and limitations of each method are discussed in depth. Potential protection tools for future microgrid are suggested at the end of this paper.
\end{abstract}

Keywords-Microgrid Protection, AC Microgrid, DC Microgrid, Protection Issues, Dual Mode Operation, Distributed Generation.

\section{INTRODUCTION}

Nowadays, distributed generation based on renewables has become a hot topic in many countries. In 2017, power generation accounts for $29.3 \%$ of total power generation in UK [1]. However, if all of these renewables are interconnected to distribution system, it would be difficult to manage these resources effectively [2]. To solve this issue, a new concept called 'microgrid' is developed.

Microgrid is a cluster of generators, loads and storage devices [3]. Generally, it can operate in two modes called grid connected mode and islanded mode. In grid connected mode, microgrid operates as a normal DG, which contributes its surplus power to the main grid. When fault occurs in utility grid, microgrid will be isolated by the breaker at point of common coupling (PCC). After that, it transfers to islanded operating mode. Based on the research in [4], fault level of microgrid varies significantly when it operates in different modes. In grid connected mode, fault current is large due to the participation of utility grid. In this case, fault can be detected and isolated easily by traditional overcurrent protection scheme. But in islanded mode, since short-circuit current drops sharply due to loss of main grid, overcurrent relay designed for grid-connected protection cannot not detect faults anymore. This problem would be more serious if most generators in microgrid are interfaced by inverters as the fault current contributed by inverter interfaced generator is limited within twice as normal loading conditions [5].
In recent years, driven by the achievement of power converter technologies, the number of DC loads, as computers and electric vehicle chargers, has gained a significant increase [6]. If all of these loads and generators are connected in traditional AC microgrid, unnecessary power losses would be caused owing to multi-stage power conversion between DC and AC. This motivates the development of DC microgrid. However, to the best knowledge of the author, protection for DC microgrid is still a big challenge due to the high magnitude fault current and lack of appropriate circuit breaker.

In this paper, existing protection issues caused by microgrid are discussed in section II. In sections III and IV, protection methods proposed in previous literatures for AC and DC microgrids are reviewed comprehensively. Moreover, the merits and demerits of listed methods are illustrated so that readers can have a better knowledge of each method. In section $\mathrm{V}$, potential tools used for the futuristic microgrid protection are suggested according to the authors' understanding. Finally, a conclusion is given in section VI.

\section{PROTECTION ISSUES}

\section{A. Bidirectional Power Flow}

When the microgrid operates in grid-connected mode, it delivers power to the grid, which causes the current in grid flowing in two directions. In this case, distribution network is evolved into an active network with bi-directional power flow. As proposed in [7], traditional overcurrent cannot distinguish forward and backward faults owing to the absence of bidirectional components. Consequently, undesired tripping might occur due to access of microgrid.

\section{B. Protection Blinding}

The circuit in Fig. 1 is employed to describe the protection blinding problem. As shown by this figure, when microgrid is connected between main source and fault location, the fault current measured by relay $\mathrm{R} 1$ will decrease owing to the current division, which shortens the relay reach of $\mathrm{R} 1$ [8]. This scenario is called protection blinding.

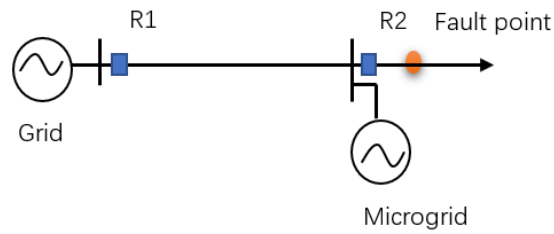

Fig. 1. Case of protection blinding

\section{Sympathetic tripping}

Sympathetic tripping is defined as the false tripping of the relay in healthy feeder due to the back-flow current from embedded microgrid [8]. For example, in Fig. 2, if sympathetic tripping occurs, relay $\mathrm{R}_{3}$ would trip for the fault at another 
line. Additionally, the probability of sympathetic tripping would increase as the microgrid on healthy feeder approaches to the busbar.

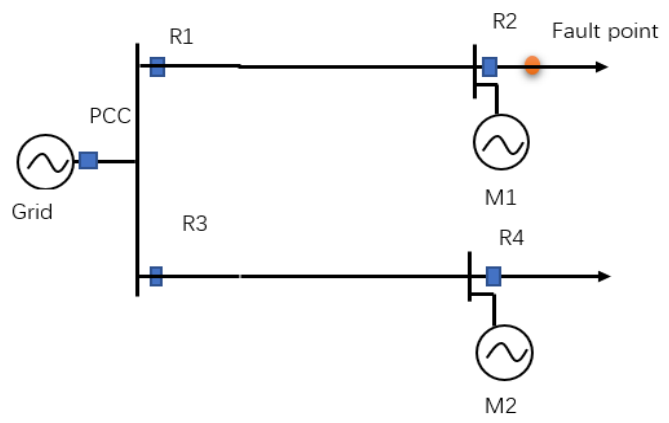

Fig. 2. Case of sympathetic tripping

\section{Varied fault level in dual mode operation and fault detection in islanded microgrid}

As aforementioned above, fault level in microgrid is determined by its operating mode, which causes the overcurrent relay designed for grid connected mode cannot provide reliable protection for microgrid [4]. Moreover, in the case of islanded microgrid, the fault current would decrease sharply, which increases the difficult of fault detection. Therefore, developing an appropriate protection scheme available for dual-mode operating conditions is an urgent task for the protection of microgrid.

\section{E. Quick capacitor discharge and high-speed protection request}

The issue proposed in this part mainly exists in DC microgrid protection. As presented in Fig. 3, when pole to pole fault occurs in cable connected with voltage source converter, the fault response of DC microgrid can be divided into three stages [9]. In the first stage, a high fault current would be introduced due to the quick discharge of capacitor and low impedance of DC cable. During this stage, fault response can be regarded as a typical RLC circuit response. The second stage is called 'freewheeling stage'. At this time, the anti-parallel diodes will provide a flowing path for fault current. Considering the safe operation of power electronic devices, all faults should be isolated before this stage. The last stage is called 'Grid feeding stage'. Normally, all faults have been cleared before second stage, therefore, this stage is not essential as previous stages. Based on the researches in [10], all DC faults should be detected and isolated within $2 \mathrm{~ms}$, which is almost 10 times faster than AC system protection. Hence, a high-speed protection scheme is compulsory for DC microgrid protection.

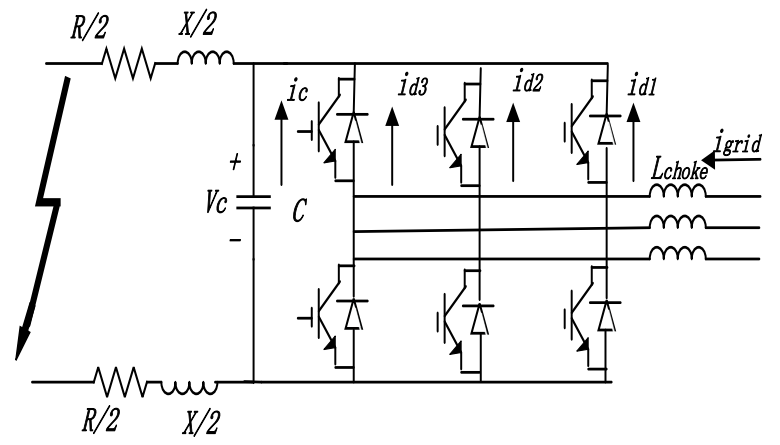

Fig. 3. Pole to pole fault in DC microgrid [9]

\section{PROTECTION METHODS FOR AC MICROGRID}

In previous literatures, many protection schemes have been proposed for AC microgrid. According to their fundamentals, these methods can be grouped as adaptive protection based, differential protection based, travelling wave based and other protective methods. The following part in this section will discuss these methods detailly. Moreover, the merits and demerits of proposed methods would be discussed so that readers can make a choice easily among these methods according to their demands.

\section{A. Adaptive protection based methods}

Adaptive protection is an automatic process where relay settings can be amended automatically to cater for the prevailing system condition so that the system can always operate in the optimal condition [11]. According to the protection logic, adaptive protection can be divided as off-line [12][13] and online protection [15][16]. In offline mode, all possible system configurations and DGs' status (on/off) would be recorded, which is used to do the offline fault analysis. The obtained result will be stored in event table. Then, based on the result in event table, appropriate relay settings would be calculated [14]. However, considering the intermittent nature of renewables and the changeable feature of microgrid, it is hard to forecast all possible fault cases in a microgrid. Therefore, the off-line mode adaptive protection is hard to be implemented in practical condition. For this reason, some scholars propose online adaptive protection where relay settings are determined by real-time system conditions. In [15], an online adaptive protection scheme is proposed to ensure the dual-mode operation of microgrid. In this scheme, relay setting is determined by equation (1). In this equation, 'mode of operation' shows the fault contribution from main grid. For example, in grid-connected mode, it will be ' 1 ' and in islanded mode, it will be 0 . In islanded mode, fault level of microgrid is dependent on the generators in the microgrid. The author utilizes a number of parameters to simulate the dynamic behaviours of DGs in different operating conditions. These behaviours including DGs' status (On/off), type (Inverter based or synchronous based) and numbers, are considered in the second part of this equation.

$\mathrm{I}_{\text {relay_set }}=\mathrm{I}_{\text {Fault_Grid }} \times$ Mode of operation +

$\sum_{\mathrm{i}=1}^{\mathrm{n}}\left(\right.$ Impact factor $\left(\mathrm{K}_{\mathrm{i}}\right) \times \mathrm{I}_{\text {Fault_ }_{\text {DG }}} \times \mathrm{DG}_{\mathrm{i}}$ status $)$

Another on-line adaptive protection is presented in [16]. Three-layer structure is employed in this scheme shown as Fig. 4. Execution layer is responsible for monitoring the primary system. Then, according to the data from execution layer, coordination layer calculates appropriate settings of relays to ensure their coordination. To avoid the frequent changes in relay setting, this scheme introduces a comparative logic in terms of operating time. This means the new relay setting would only be employed when the operating time calculated from new settings is shorter than previous one. Management layer is at the top of this scheme, which deals with the relationship between active network management and relay coordination. In general, adaptive protection is one of the most promising methods to overcome the protection issues raising from varied fault level since it can reflect the dynamic behaviours of power system and DGs. In adaptive protection, communication channels are commonly employed, which increases the cost of protection 
scheme. On the other hand, it would be dangerous if a communication failure occurs in the grid.

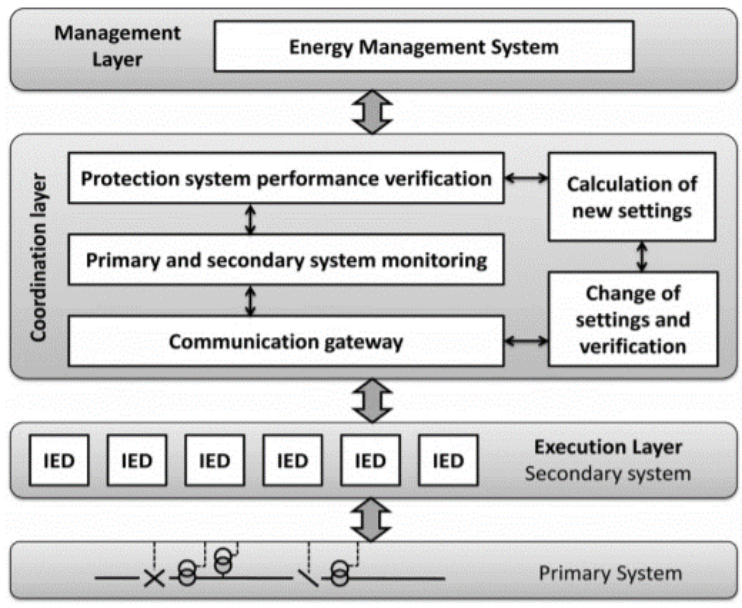

Fig. 4. Three-layer adaptive protection [16]

\section{B. Protection scheme based on differential scheme}

Differential protection is another commonly utilized method for microgrid protection. The basic operating fundamentals and parameter setting procedure of differential protection are explained in [17]. As proposed in this paper, current differential protection is less sensitive to fault current variation, bi-directional power flow, DGs status and the changes of system configuration. Microgrid protection system based on differential algorithm is presented in [18]. In this protection system, all relays are digital relay equipped with communication capability, which can sample the current-wave form at more than 16 samples per cycle. These relays are installed at the both ends of protected feeders and sample the current flowing through them. If the current differences captured by two end relays are over than the tripping threshold, relay would send a tripping signal to open the respective breakers. Through validation, the performance of this system is satisfied. However, the cost of this system would be a barrier of practical use as it requires the use of synchronous measurement and communication channels. To further decrease the investment of differential protection, an optimal differential protection scheme is presented in [19]. The author divides the total cost of differential protection scheme as two parts, including protection devices (relay, breaker, CT, VT) and additional cost caused by power interruption. Meanwhile, the author believes there is no need to put digital relays at both ends of feeders, which can be replaced by cheap sensors. Based on the results of a case study in this paper, this method can save around half price comparing with scheme in [18]. However, because there is no supported result to evaluate the protection performance of this optimized differential system, it is uncertainty whether this system can achieve the same protection performance as before. Differential scheme relying on the spectral energy at both ends is discussed in [20]. In this paper, current measured at two ends is transformed to frequency domain through ' $\mathrm{S}$ transform' [21] to calculate the spectral energy. The tripping logic is similar with current based differential scheme. Relay would trip when the energy difference is larger than defined threshold. In this method, a small synchronous error is allowed and it can also detect the fault with high impedance. But as proposed in this paper, threshold selection is a tough job in this scheme, which is determined by many factors including fault impedance, operating modes, etc.

\section{Other protective methods}

1) Voltage based method

Considering the suppressed fault current in islanded microgrid, some scholars try to develop the protection schemes by using system voltage. An example is presented in [3]. In this scheme, all generators are interfaced by using three-phase inverter. The fault is detected by measuring the voltage disturbance of the $\mathrm{q}$ axis, which is a DC variable obtained by using Park Transform [22]. Voltage disturbance $V_{D I S T}$ is defined as equation (2). The reason why we only use voltage in $\mathrm{q}$ axes is that voltage at $\mathrm{d}$ axes has been locked to zero by Phase Lock Loop (PLL). In normal loading or balanced faults condition, $V_{q r}$ would be a pure DC wave and $V_{D I S T}$ in (2) would be zero. While, in the case of a single line to ground fault, $V_{D I S T}$ is an oscillating signal varying between zero and maximum value. When the system experiences phase to phase fault, it would be a dc voltage with ac ripple. Hence, through observing the features of $V_{D I S T}$, fault condition and types can be detected. However, same as energy-based differential protection, a threshold is required to determine tripping of relays. If this threshold is too large, this protection scheme would not be sensitive to detect faults especially in high impedance fault condition (HIFs). But if the value is too low, undesired tripping would be initiated by normal disturbance such as mode switching of microgrid.

$$
V_{D I S T}=V_{q r e f}-V_{q r}
$$

\section{2) Total harmonic distortion based method}

Total harmonic distortion (THD) is utilized in [23] for the protection of converter dominated microgrid. This protection scheme consists two stages including fault type identification and fault localization. In the first stage, fault type is defined by measuring the voltage with fundamental frequency and THD of each phase. For example, if fundamental voltage decreases and THD increases in terms of phase A, it can be concluded that phase A to ground fault occurs in the network. Additionally, as the value of THD increases as the decrease of distance, the faulted distance can be estimated. While according to [24], the performance of THD-based fault detection is determined on the fault current limiting strategy and implementing frame (SYRF, STRF, NARF) of the voltage source converter (VSC). As proposed by this article, THD based method would fail to detect faults when hybrid reference frame limiting (HRFL) strategy is implemented. Furthermore, as a threshold is also necessary in THD protection scheme, the similar problem will experience as before.

\section{3) Phasor Based method}

In [25], a protection scheme based on the phase angle variation of postfault bus voltage and current is developed. The working procedure of this scheme is shown as Fig. 5. The variable $R$ and $\Delta \varphi_{i j}$ are defined by equation (3) and (4) respectively. Both variables are used as index criteria for fault detection and localization.

$$
\begin{gathered}
R=F_{i} / F_{n} \\
\Delta \varphi_{i j}=\arg \left(\Delta \dot{U}_{l}\right)-\arg \left(\Delta \dot{i}_{l \jmath}\right)
\end{gathered}
$$


Where $F_{i}$ and $F_{n}$ are defined as $\frac{\Delta I_{0}+\Delta I_{2}}{\Delta I_{1}}$ and $\frac{I_{0}+I_{2}}{I_{1}}$. $\Delta I_{0}, \Delta I_{1}, \Delta I_{2}$ and $I_{0}, I_{1}, I_{2}$ stand for the sequence current components during fault and normal cases. $\Delta \dot{U}_{i}$ and $\Delta \dot{I}_{i j}$ are the phasor change of pre-fault and postfault voltage at bus $i$ and phasor change of current in the feeder connected by bus $\mathrm{i}$ and bus $\mathrm{j}$.

From Fig. 5, it can be concluded that if fault occurs in the microgrid, the value of $\Delta \varphi_{i j}$ should be in the range of $-180^{\circ}$ and $0^{\circ}$. As a branch feeder is only connected one upstream bus, only the phasor variation of the upstream bus should be considered. However, the main feeder connects two buses, hence, phasor variations at two buses are required. There is no need of communication in this system and it is reliable in the case of high impedance fault. However, it can only protect the grid-connected microgrid, which is the main drawback of this scheme.

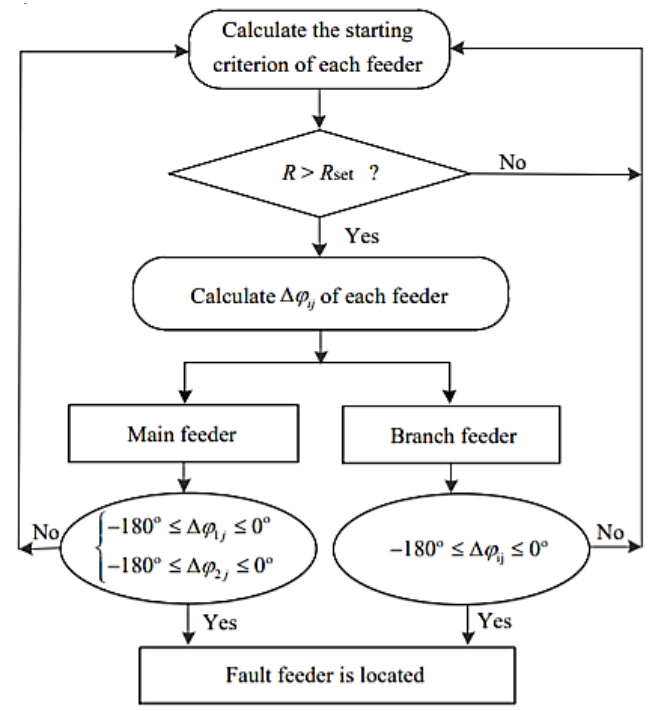

Fig. 5. Fault detection based on variation of voltage and current [25]

\section{Travelling wave based method}

Travelling wave method has been widely used for the fault localization in high voltage transmission system, especially in HVDC system [26]. However, owing to the limiting factors as high sampling frequency, noise disturbance, etc [27], it is rarely used as microgrid protection. In [28], an improved travelling wave based protection scheme is established for fault detection in inverter dominated microgrid. In this paper, improved mathematical morphology (MM) is implemented to capture the polarity of initial current travelling wave. More detail information about MM method can be founded in [29]. When an internal fault occurs, the polarity of initial wave captured at both ends would be the same, while it would be opposite under the case of external faults. In this paper, ' 1 ' and ' -1 ' are defined as positive and negative polarity. If the product of both polarities is positive, this indicates internal fault occurs and the relay will be initiated. According to validated result, this protection scheme has a good performance on noise disturbance and high impedance fault condition. Moreover, it is not influenced by the mode switching and transient disturbance of microgrid. However, the main limitation of travelling wave based method is the high requirement of sampling frequency especially when the feeder length is too short.

\section{DC MICROGRID PROTECTION}

Except for experiencing the common protection issues with AC microgrid, DC microgrid is also threatened by a high magnitude fault current caused by the quick discharge of capacitor [30]. To avoid the physical damage of power electronic devices (IGBTs, Diodes), fast speed protection is required. In this section, several schemes for DC microgrid protection would be presented.

\section{A. Fault detection based on the rate of change of volatage and current}

In DC system, owing to the fast discharging of capacitor and low cable impedance, fault current can reach an extremely large value in transient time period. This large current would cause physical damage to electronic devices in DC microgrid. To avoid this damage, all faults in DC microgrid should be isolated within $2 \mathrm{~ms}$. Derivations of current and voltage $\left(d_{i} / d t, d_{v} / d t\right)$ are commonly employed as their responses to fault are more sensitive than pure current and voltage-based methods. A fault detection method based on $d_{i} / d t$ is discussed in [31]. In this scheme, the tripping threshold $\Delta \mathrm{i}$ is presented as equation (5), which is deduced from the RLC fault response.

$$
\Delta i \cong \frac{V_{C(0)}}{L} \Delta t
$$

Given the fact that current during fault is much larger than it in normal loading condition, the initial condition of current is assumed as zero in this equation. Whilst, it is clear that this tripping threshold is determined by the value of $\Delta t$, which is the desired tripping time controlled by users. Hence, through varying $\Delta t$, the coordination between relays can be achieved. In [32], a protection scheme based on the difference between predicted and measured voltage is proposed. This predicted voltage is obtained by using Quadratic Model [33]. In Quadratic Model, the predicted voltage is determined by previous sampling points of voltage, therefore any variation of voltage caused by fault would introduce an obvious change in predicted wave. If the difference between predicted and measured voltage is over than defined threshold, relay would trip. Generally, methods based on rate of change of current and voltage are sensitive enough for fault detection. However, this type of method is easily disturbed by the measuring noise and normal transient behaviour of power system [34].

\section{B. Differential based protection scheme}

Comparing with non-unit scheme, differential protection is believed as one of the best solutions for DC microgrid [30]. As proposed in [35], there are two main issues for the practical use of differential protection. The first one is the error from synchronous measurement of ended current. The second one is the strict requirement for operating speed. Nowadays, with the achievement of communication and synchronous sampling technologies, this error can be limited within $\perp_{-} 1 \mu \mathrm{s}$ by using GPS time sampling, which is acceptable in DC microgrid protection. On the other hand, in AC system, the phasor and current magnitude are both measured by relays, which causes a time delay. While in DC system, only current magnitude is required and the output of measured device is in the form of digital signal, which does not need the process of analogue to digital conversion. For this reason, the time of decision making process of DC protection relay would decrease significantly. To validate the feasibility of above conclusions, a current based differential scheme is built in this 
paper and it has been tested by hardware. Depending on the testing results, all internal faults can be isolated within $2 \mathrm{~ms}$. Another example is presented in [36]. In this paper, the superimposed current is used, which is defined as the vector difference of postfault and prefault current. The positive direction is given by Fig. 6. When internal fault occurs, superposed currents measured by relays at both ends are always positive. On the contrary, in term of external fault or normal loading condition, the superposed current at one end would be negative. Hence, if the point made by superposed currents at both ends lies in the first quadrant, the protective relay would be tripped. In differential scheme, more relays and measuring equipment are needed, therefore, the cost of this would be much higher than non-unit protection.

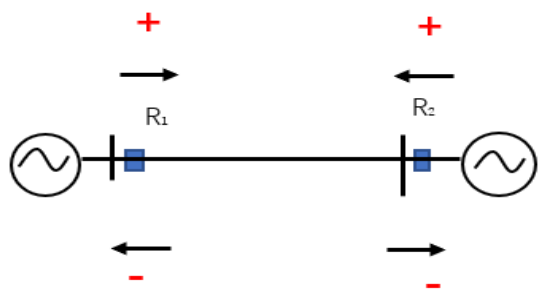

Fig. 6. Direction reference of positive and negative current flow

\section{DISCUSSION ON POTENTIAL PROTECTION SOLUTIONS}

In the future, varying fault levels will become more obvious in microgrid due to large penetration of renewables. Under this background, the conventional protection scheme with a fixed setting group would not be feasible. Therefore, adaptive protection is likely to be one of the most attractive solutions for AC microgrid. Moreover, driven by the developments of new technologies (such as $5 \mathrm{G}$ ), fast and reliable communication is becoming achievable. This provides a good opportunity for the implementation of adaptive protection in AC microgrid. On the other hand, considering the increased penetration of power electronic inverter in microgrid, fault detection in islanded microgrids will be another challenge owing to the suppressed current magnitude. In this case, some signal processing tools such as wavelet transform [37] or mathematical morphology [28] can be used to detect fault in the microgrid.

In DC microgrids, fault current can increase to a very large value within a very short period of time. If the fault is not isolated rapidly (approximately within $2 \mathrm{~ms}$ ), it is likely to cause damage to power electronic devices. Under this case, differential protection becomes a valid choice for DC microgrid protection as demonstrated in [30]. Comparing to $\mathrm{AC}$ system, there is no natural current zero-crossing in DC system, which makes the circuit breaker designed for $\mathrm{AC}$ system not valid anymore. Although solid-state circuit breaker can facilitate fast isolation, the high cost is likely to be prohibitive for the wide use in the industry [38]. Different with the aforementioned ideas, another way for DC microgrid protection limits the increment of fault current by introducing additional devices rather than speeding up the protection [39].

Hybrid AC/DC microgrid overcomes the drawback of pure AC or DC microgrid. However, the protection for hybrid $\mathrm{AC} / \mathrm{DC}$ microgrid is rarely discussed in technical literature. Hence, an innovative protection scheme for hybrid AC/DC microgrid should be developed in the future [40].

\section{CONCLUSION}

In the future power system, a large number of renewables will be utilized. Microgrid provides an effective way to interconnect these renewables. However, owing to the varied fault level and low fault current in islanded microgrid, traditional protection methods are not feasible. Comparing with AC microgrid, DC microgrid protection is threatened by the high magnitude fault current and lack of current zerocrossing. In this paper, a comprehensive review of existing issues and protection methods for microgrid is presented. Given the dynamic behaviour of microgrid and suppressed fault current level, adaptive protection and fault detection scheme assisted by signal processing tools are suggested. For DC microgrid, differential protection is regarded as one of the most promising methods due to its fast operating speed. Moreover, circuit breakers available for commercial use should be designed in the future. Finally, this paper points out more efforts should be paid for the protection of hybrid AC/DC microgrid.

\section{REFERENCES}

[1] GOV.UK. (2019). UK energy in brief 2018. [online] Available at: https://www.gov.uk/government/statistics/uk-energy-in-brief-2018.

[2] E. Sortomme, J. Ren and S. S. Venkata, "A differential zone protection scheme for microgrids," 2013 IEEE Power \& Energy Society General Meeting, Vancouver, BC, 2013, pp.

[3] Al-Nasseri, H., Redfern, M.A., Li, F.: 'A voltage based protection for microgrids containing power electronic converters'. Proc. IEEE Power Engineering Society General Meeting, Montreal, Canada, June 2006, pp. 1-5.

[4] U. Maqbool and U. A. Khan, "Fault current analysis for grid-connected and Islanded microgrid modes," 2017 13th International Conference on Emerging Technologies (ICET), Islamabad, 2017, pp. 1-5.

[5] Manditereza, P. and Bansal, R. (2018). Review of technical issues influencing the decoupling of DG converter design from the distribution system protection strategy. IET Renewable Power Generation, 12(10), pp.1091-1100.

[6] C. Li, P. Rakhra, P. Norman, G. Burt and P. Clarkson, "Metrology requirements of state-of-the-art protection schemes for DC microgrids," in The Journal of Engineering, vol. 2018, no. 15, pp. 987992, 102018

[7] A. Jalilian, M. T. Hagh and S. M. Hashemi, "An Innovative Directional Relaying Scheme Based on Postfault Current," in IEEE Transactions on Power Delivery, vol. 29, no. 6, pp. 2640-2647, Dec. 2014

[8] V. A. Papaspiliotopoulos, G. N. Korres, V. A. Kleftakis and N. D. Hatziargyriou, "Hardware-In-the-Loop Design and Optimal Setting of Adaptive Protection Schemes for Distribution Systems With Distributed Generation," in IEEE Transactions on Power Delivery, vol. 32, no. 1, pp. 393-400, Feb. 2017.

[9] J. Yang, J. E. Fletcher and J. O'Reilly, "Short-Circuit and Ground Fault Analyses and Location in VSC-Based DC Network Cables," in IEEE Transactions on Industrial Electronics, vol. 59, no. 10, pp. 3827-3837, Oct. 2012.

[10] R. Li, L. Xu and L. Yao, "DC Fault Detection and Location in Meshed Multiterminal HVDC Systems Based on DC Reactor Voltage Change Rate," in IEEE Transactions on Power Delivery, vol. 32, no. 3, pp. 1516-1526, June 2017.

[11] Phadke and J. Thorp, Computer Relaying for Power Systems, 2nd ed. Hoboken, NJ, USA: Wiley, 2009.

[12] S. M. Brahma and A. A. Girgis, "Development of adaptive protection scheme for distribution systems with high penetration of distributed generation," 2003 IEEE Power Engineering Society General Meeting (IEEE Cat. No.03CH37491), Toronto, Ont., 2003, pp. 2083-2083.

[13] P. Mahat, Z. Chen, B. Bak-Jensen and C. L. Bak, "A Simple Adaptive Overcurrent Protection of Distribution Systems With Distributed Generation," in IEEE Transactions on Smart Grid, vol. 2, no. 3, pp. 428-437, Sept. 2011.

[14] A. Oudalov, A. Fidigatti, "Adaptive network protection in microgrids," Int J Distrib Energy Resour, 4 (3) (2009). 
[15] W. L. T. Peiris, W. H. Eranga, K. T. M. U. Hemapala and W. D. Prasad, "An Adaptive Protection Scheme for Small Scale Microgrids Based on Fault Current Level," 2018 2nd International Conference on Electrical Engineering (EECon), Colombo, 2018, pp. 64-70.

[16] F. Coffele, C. Booth and A. Dyśko, "An Adaptive Overcurrent Protection Scheme for Distribution Networks," in IEEE Transactions on Power Delivery, vol. 30, no. 2, pp. 561-568, April 2015.

[17] M. Dewadasa, A. Ghosh and G. Ledwich, "Protection of microgrids using differential relays," AUPEC 2011, Brisbane, QLD, 2011, pp. 16.

[18] E. Sortomme, S. S. Venkata and J. Mitra, "Microgrid Protection Using Communication-Assisted Digital Relays," in IEEE Transactions on Power Delivery, vol. 25, no. 4, pp. 2789-2796, Oct. 2010.

[19] E. Sortomme, J. Ren and S. S. Venkata, "A differential zone protection scheme for microgrids," 2013 IEEE Power \& Energy Society General Meeting, Vancouver, BC, 2013, pp. 1-5.

[20] S. R. Samantaray, G. Joos and I. Kamwa, "Differential energy based microgrid protection against fault conditions," 2012 IEEE PES Innovative Smart Grid Technologies (ISGT), Washington, DC, 2012, pp. 1-7.

[21] Mansinha, L., Stockwell, R. and Lowe, R. (1997). Pattern analysis with two-dimensional spectral localisation: Applications of twodimensional S transforms. Physica A: Statistical Mechanics and its Applications, 239(1-3), pp.286-295.

[22] Yazdani, A. and Iravani, R. (2010). Voltage-Sourced Converters in Power Systems. Hoboken: John Wiley \& Sons.

[23] H. Al-Nasseri and M. A. Redfern, "Harmonics content based protection scheme for Micro-grids dominated by solid state converters," 2008 12th International Middle-East Power System Conference, Aswan, 2008, pp. 50-56.

[24] I. Sadeghkhani, M. E. Hamedani Golshan, A. Mehrizi-Sani, J. M. Guerrero and A. Ketabi, "Transient Monitoring Function-Based Fault Detection for Inverter-Interfaced Microgrids," in IEEE Transactions on Smart Grid, vol. 9, no. 3, pp. 2097-2107, May 2018.

[25] F. Zhang and L. Mu, "A Fault Detection Method of Microgrids with Grid-Connected Inverter Interfaced Distributed Generators Based on the PQ Control Strategy," in IEEE Transactions on Smart Grid.

[26] S. Lin, Z. Y. He, X. P. Li and Q. Q. Qian, "Travelling wave timefrequency characteristic-based fault location method for transmission lines," in IET Generation, Transmission \& Distribution, vol. 6, no. 8, pp. 764-772, August 2012.

[27] A. Zidan et al., "Fault Detection, Isolation, and Service Restoration in Distribution Systems: State-of-the-Art and Future Trends," in IEEE Transactions on Smart Grid, vol. 8, no. 5, pp. 2170-2185, Sept. 2017.

[28] X. Li, A. Dyśko and G. M. Burt, "Traveling Wave-Based Protection Scheme for Inverter-Dominated Microgrid Using Mathematical Morphology," in IEEE Transactions on Smart Grid, vol. 5, no. 5, pp. 2211-2218, Sept. 2014.
[29] S. Gautam and S. M. Brahma, "Overview of mathematical morphology in power systems - A tutorial approach," 2009 IEEE Power \& Energy Society General Meeting, Calgary, AB, 2009, pp. 1-7.

[30] S. D. A. Fletcher, P. J. Norman, S. J. Galloway, P. Crolla and G. M. Burt, "Optimizing the Roles of Unit and Non-unit Protection Methods Within DC Microgrids," in IEEE Transactions on Smart Grid, vol. 3, no. 4, pp. 2079-2087, Dec. 2012.

[31] A. Meghwani, S. C. Srivastava and S. Chakrabarti, "A new protection scheme for DC microgrid using line current derivative," 2015 IEEE Power \& Energy Society General Meeting, Denver, CO, 2015, pp. 1-5.

[32] A. Meghwani, S. Chakrabarti and S. C. Srivastava, "A fast scheme for fault detection in DC microgrid based on voltage prediction," 2016 National Power Systems Conference (NPSC), Bhubaneswar, 2016, pp. 1-6.

[33] A. Eisenberg and P.Pugliese, "Exact inversion of a class $\mathrm{f}$ vandermonde matrices," in Proc. 5th SIAM conference on applied algebra, June 1994.

[34] C. Li, P. Rakhra, P. Norman, P. Niewczas, G. Burt and P. Clarkson, "Practical computation of $\mathrm{di} / \mathrm{dt}$ for high-speed protection of DC microgrids," 2017 IEEE Second International Conference on DC Microgrids (ICDCM), Nuremburg, 2017, pp. 153-159.

[35] S. D. A. Fletcher, P. J. Norman, K. Fong, S. J. Galloway and G. M. Burt, "High-Speed Differential Protection for Smart DC Distribution Systems," in IEEE Transactions on Smart Grid, vol. 5, no. 5, pp. 26102617, Sept. 2014.

[36] R. Mohanty and A. K. Pradhan, "A Superimposed Current Based Unit Protection Scheme for DC Microgrid," in IEEE Transactions on Smart Grid, vol. 9, no. 4, pp. 3917-3919, July 2018.

[37] P. Kanakasabapathy and M. Mohan, "Digital protection scheme for microgrids using wavelet transform," 2015 IEEE International Conference on Electron Devices and Solid-State Circuits (EDSSC), Singapore, 2015, pp. 664-667.

[38] Xiang, W., Hua, Y., Wen, J., Yao, M. and Li, N. (2014). Research on fast solid state DC breaker based on a natural current zero-crossing point. Journal of Modern Power Systems and Clean Energy, 2(1), pp.30-38.

[39] C. Peng, J. Wen, Y. Wang, J. Wu and X. Chen, "Potential Use of Fault Current Limiter in VSC Based DC Transmission Systems," 2012 AsiaPacific Power and Energy Engineering Conference, Shanghai, 2012, pp. 1-4.

[40] R. A. Kaushik and N. M. Pindoriya, "A hybrid AC-DC microgrid: Opportunities \& key issues in implementation," 2014 International Conference on Green Computing Communication and Electrical Engineering (ICGCCEE), Coimbatore, 2014, pp. 1-6. 